\section{PKS}

PUBLIC

KNOWLEDGE

PROJECT

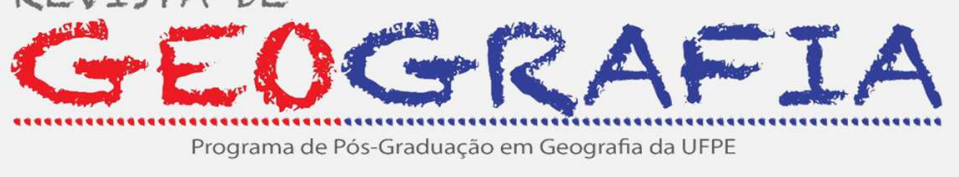

https://periodicos.ufpe.br/revistas/revistageografia
OJS

OPEN

JOURNAL

SYSTEMS

\title{
ÚLTIMOS REFÚGIOS DA ONÇA-PARDA (PUMA CONCOLOR) NO BRASIL: ÁREAS PRIORITÁRIAS PARA A CONSERVAÇÃO SOB A PERSPECTIVA DA MODELAGEM DE DISTRIBUIÇÃO GEOGRÁFICA
}

\author{
Felipe Francisco Gomes-Silva ${ }^{1 *}$, Edson Silva Barbosa Leal ${ }^{2}$
}

${ }^{1}$ Analista ambiental do Instituto de Tecnologia de Pernambuco, Diretoria Executivo-Comercial, Escritório de Projetos. Av. Prof. Luiz Freire, 700. Cidade Universitária, Recife - PE. E-mail: f.lipegs@ gmail.com. https://orcid.org/0000-00032058-6566

${ }^{2}$ Programa de Pós-Graduação em Biologia Animal, Universidade Federal de Pernambuco, Centro de Biociências, Departamento de Zoologia, Laboratório de Ciência Aplicada à Conservação da Biodiversidade, Rua Nelson Chaves, s/n, Cidade Universitária, Recife - PE. E-mail: edsonsbl@yahoo.com.br. https://orcid.org/0000-0002-6577-2330

* Autor para correspondência.

Artigo recebido em 07/06/2020 e aceito em 21/02/2021

\begin{abstract}
RESUMO
A modelagem de distribuição geográfica de espécies é uma importante ferramenta da biogeografia que atende ações conservacionistas ou permite abordagens teóricas sobre a distribuição das espécies. No presente estudo, modelamos a distribuição geográfica da onça-parda (Puma concolor) no Brasil através de quatro algoritmos (Environmental Distance, GARP, Maximum Entropy, BioClim). Os dados de ocorrência da onça-parda foram obtidos a partir da plataforma speciesLink e mediante revisão de literatura. Foram selecionadas as variáveis bioclimáticas disponíveis na homepage do WorldClim (www.worldclim.com). Ao todo, foram obtidos 280 registros de onça-parda no Brasil. Os modelos gerados indicam que a Caatinga, Pantanal, Mata Atlântica (ao norte do rio São Francisco) e Pampa representam os biomas com menor adequabilidade ambiental. Em contrapartida, a porção de Mata Atlântica localizada na região sudeste sob influência da Serra do Mar e limítrofe ao Cerrado, representa uma das áreas com maior probabilidade de abrigar populações de Puma concolor. Cerrado e Amazônia também apresentaram média e alta adequabilidade ambiental. Essas informações indicam a necessidade do estabelecimento de novas áreas protegidas onde a probabilidade de ocorrência foi inferior ao esperado. Adicionalmente, medidas que minimizem os impactos da caça e aumentem a conectividade da paisagem devem ser fortalecidas no sentido de assegurar a persistência dessas populações em médio e longo prazo.
\end{abstract}

Palavras-chave: Puma concolor. Distribuição geográfica. Onça-parda. Modelagem. Áreas prioritárias.

\section{PUMA'S (PUMA CONCOLOR) LAST REFUGEES IN BRAZIL: PRIORITY AREAS FOR CONSERVATION FROM THE PERSPECTIVE OF GEOGRAPHICAL MODELLING}

\begin{abstract}
The modeling of geographic distribution of species is an important biogeography tool that meets conservation actions or allows theoretical approaches on the distribution of species. In this study, we modeled the geographic distribution of the puma (Puma concolor) in Brazil through four algorithms (Environmental Distance, GARP, Maximum Entropy, BioClim). The occurrence data from puma were obtained from the speciesLink platform and through a literature review. Bioclimatic
\end{abstract}


variables available on the home page of WorldClim (www.worldclim.com) were selected. Overall, 280 puma records were obtained in Brazil. The models indicate that the Caatinga, Pantanal, Atlantic Forest (north of San Francisco river) and Pampa biomes represent less environmental suitability. In contrast, the Atlantic portion located in the southeast under the influence of the Serra do Mar and neighboring Cerrado, is one of the areas most likely to harbor populations of Puma concolor. Cerrado and Amazon also had medium and high environmental suitability. This information indicates the need for establishing new protected areas where the probability was lower than expected. In addition, measures to minimize the impacts of hunting and increase landscape connectivity should be strengthened to ensure the persistence of these populations in the medium and long terms.

Keywords: Puma concolor. Geographic distribution. Cougar. Modelling. Priority areas.

\section{INTRODUÇÃO}

Mamíferos de médio e grande porte integram processos-chave para o equilíbrio dos ecossistemas, pois atuam como importantes dispersores, predadores, além de representarem espécies guarda-chuva, uma vez que suas demandas ecológicas asseguram a conservação de toda uma cadeia de biodiversidade em níveis tróficos inferiores (FOLEY, 1982, ROBERGE e ANGELSTAM, 2004). Essas espécies geralmente ocorrem em baixas densidades e apresentam amplas distribuições geográficas (EISENBERG, 1980; GITTLEMAN e HARVEY, 1982), convertendo-se em interessantes modelos para a predição dos impactos de alterações ambientais sobre a biodiversidade em longo prazo.

A onça-parda (Puma concolor), em algumas regiões também denominada onça vermelha ou suçuarana, é um carnívoro conhecido pela sua plasticidade em termos de distribuição geográfica, uma vez que ocorre tanto em regiões temperadas quanto em florestas tropicais (CURRIER, 1983). Terborgh $(1988,1992,2000)$, aponta a onça-parda como importante predador-chave em florestas tropicais, ao lado da onça-pintada (Panthera onca), regulando populações de mamíferos de médio porte (MORENO et al., 2011; SCOGNAMILLO et al., 2003).

Nesse contexto, a compreensão dos processos que determinam a distribuição e ocorrência de tais populações se torna um objetivo primário visando a proposição de medidas para a conservação dessas espécies (GIANNINI, 2012). Ademais, a identificação de áreas com alta adequabilidade de habitats também é uma importante ferramenta para o estabelecimento das bases para estudos de campo e para o planejamento de iniciativas de conservação (THORN et al., 2009). Para isso, dados insuficientes ou imprecisos sobre a distribuição de uma determinada espécie podem ser modelados a partir de algoritmos computacionais fornecendo indicativos da probabilidade de ocorrência de uma espécie em determinada região a partir de um conjunto de variáveis ambientais (ENGLER et al., 2004). Essa técnica é conhecida como modelagem de distribuição de espécies, alternativamente 
denominada modelagem de nicho ecológico e tem sido extensivamente adotada em estudos que objetivam avaliar e predizer a ocorrência de espécies sob diferentes condições ambientais (GUISAN e THUILLER, 2005).

A modelagem da distribuição de uma espécie assume que a sua ocorrência em determinada região é resultado da combinação de três elementos básicos: (i) um conjunto de condições abióticas (cenopoéticas) favoráveis, incluindo variáveis climáticas e do ambiente físico; (ii) as variáveis bióticas (ou bionômicas) que resultam da interação com outras espécies e que modificam sua capacidade de manter populações viáveis; (iii) a capacidade de movimento dos indivíduos de uma espécie, correspondendo às regiões geográficas que estão acessíveis a uma espécie em determinado intervalo de tempo. Essas condições bióticas (B), abióticas (A) e referentes ao movimento dos indivíduos (M), podem ser representadas através do diagrama BAM, cuja intersecção representa o nicho realizado da espécie, ou seja, uma área restrita dentro de uma ampla região ocupável pela espécie (o nicho fundamental) onde ela é superior aos seus competidores e pode persistir (PETERSON et al., 2011).

Esse conceito de nicho fundamental é definido por Hutchinson (1957) como o hipervolume de variáveis ambientais, ou seja, cada ponto ao qual corresponde um estado do ambiente que permite às espécies existirem indefinidamente. Assim, os dados de ocorrência das espécies são tão importantes quanto as variáveis ambientais associadas que serão avaliadas nos modelos de distribuição. Diante disso, o presente estudo tem como propósito modelar a distribuição geográfica da onça-parda através de diferentes cenários climáticos tomando como base as atuais informações disponíveis quanto à sua ocorrência.

\section{MATERIAL E MÉTODOS}

\section{Dados de ocorrência}

As informações referentes à ocorrência geográfica de $P$. concolor foram obtidas mediante acesso aos dados disponíveis nas bases do CRIA (Centro de Referência em Informação Ambiental), plataforma speciesLink (CRIA, 2015). A essas informações foram acrescentados dados obtidos mediante revisão de literatura que confirmassem a ocorrência de $P$. concolor no país. Os dados foram compilados considerando-se os registros realizados no período de 2000 a 2015. Para tanto, foram incluídas as coordenadas geográficas de publicações indexadas disponíveis nas bases de dados do 
Google Acadêmico (www.scholar.google.com), Scielo (www.scielo.br/), Scopus (www.scopus.com) e Web of Science (www.webofknowledge.com).

Seleção de variáveis

Para a modelagem da distribuição de $P$. concolor foram pré-selecionadas as 19 camadas da variável climática Bioclim (disponível em https://www.worldclim.org/data/worldclim21.html) a 2,5 minutos de arco (aproximadamente $4,6 \mathrm{~km}^{2}$ de resolução espacial). Essas variáveis foram consideradas adequadas para as modelagens tendo em vista os objetivos do estudo e a área de vida da

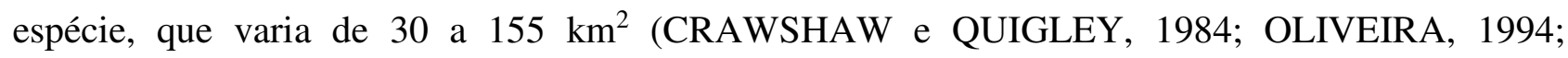
PENTEADO, 2012) (Tabela 1). Nesse sentido, a fim de eliminar variáveis altamente correlacionadas, foram realizadas análises de colinearidade descartando-se variáveis com coeficiente de correlação superior a 0,75 (BUENO, 2012).

TABELA 1 - DESCRIÇÃO DAS VARIÁVEIS CLIMÁTICAS PRÉ-SELECIONADAS PARA AS MODELAGENS DE DISTRIBUIÇÃO DE P. CONCOLOR.

\begin{tabular}{|c|c|c|}
\hline Código & $\begin{array}{l}\text { Variáve } \\
\text { l }\end{array}$ & Descrição \\
\hline 1 & bio_1 & Temperatura média anual \\
\hline 2 & bio_2 & Amplitude térmica diária média \\
\hline 3 & bio_3 & Isotérmica (bio2 / bio7) \\
\hline 4 & bio_4 & Sazonalidade da temperatura (desvio-padrão) \\
\hline 5 & bio_5 & Temperatura máxima do mês mais quente \\
\hline 6 & bio_6 & Temperatura mínima do mês mais frio \\
\hline 7 & bio_7 & Amplitude térmica anual (bio5 - bio6) \\
\hline 8 & bio_8 & Temperatura média do trimestre mais úmido \\
\hline 9 & bio_9 & Temperatura média do trimestre mais seco \\
\hline 10 & bio_10 & Temperatura média do trimestre mais quente \\
\hline 11 & bio_11 & Temperatura média do trimestre mais frio \\
\hline 12 & bio_12 & Precipitação anual \\
\hline 13 & bio_13 & Precipitação do mês mais úmido \\
\hline 14 & bio_14 & Precipitação do mês mais seco \\
\hline 15 & bio_15 & Sazonalidade da precipitação (coeficiente de variação) \\
\hline 16 & bio_16 & Precipitação do trimestre mais úmido \\
\hline 17 & bio_17 & Precipitação do trimestre mais seco \\
\hline 18 & bio_18 & Precipitação do trimestre mais quente \\
\hline 19 & bio_19 & Precipitação do trimestre mais frio \\
\hline
\end{tabular}

Modelagem dos dados

Para a modelagem da distribuição de $P$. concolor as variáveis climáticas selecionadas foram combinadas aos seguintes algoritmos de modelagem: (i) Bioclim, modelo que assume que a 
abundâncias das espécies é determinada por limites climáticos (NIX, 1986); (ii) Environmental Distance, algoritmo genérico que se baseia nas métricas de dissimilaridade ambiental (CARPENTER et al., 1993); (iii) GARP, o algoritmo genético que cria modelos de nicho ecológico para as espécies, descrevendo condições ambientais sob as quais as espécies podem manter populações (STOCKWELL e NOBLE, 1992; STOCKWELL, 1999; STOCKWELL e PETERS, 1999); e (iv) Maxent, que usa o princípio da máxima entropia sobre dados de presença-ausência para estimar um conjunto de funções que relacionam variáveis ambientais e adequabilidade do habitat no sentido de aproximar o nicho das espécies e sua respectiva distribuição geográfica (ELITH et al., 2011; PHILLIPS et al., 2006; PHILLIPS e DUDÍK, 2008).

Os produtos resultantes da modelagem foram interpretados com auxílio de um sistema de informação geográfica (pacote ArcMap disponível na suíte ArcGIS versão 10.7.1). A avaliação de desempenho dos modelos preditivos foi realizada analisando-se a área sob a curva ROC (Receiver Operating Characteristic) denominada (AUC - Area Under the Curve) e fornecida no software OpenModeller. Essa métrica tem sido amplamente adotada na avaliação de modelos preditivos de distribuição (CUYCKENS et al., 2015; FENG e PAPEŞ, 2015; PHILLIPS et al., 2006) e indica a capacidade do modelo obtido em discriminar presenças e ausências nas classificações.

As modelagens foram conduzidas com auxílio do software de código aberto OpenModeller Desktop na versão 1.1.0. As análises estatísticas foram realizadas por meio do pacote SPSS 16 (SPSS Inc., Chicago, 2005). Todas as análises foram executadas tomando-se como padrão o sistema de coordenadas geográficas WGS 1984 em grau decimal.

\section{RESULTADOS}

Foram obtidos 131 registros de $P$. concolor a partir da base de dados do speciesLink, aos quais se somaram 149 registros obtidos mediante revisão de literatura, perfazendo 280 registros da espécie no Brasil. Esses registros produziram o atual mapa de ocorrência da onça-parda no Brasil, cujos pontos foram utilizados nas modelagens (Figura 1). 
FIGURA 1 - REGISTROS DE $P$. CONCOLOR PARA O BRASIL, INCLUINDO REGISTROS OBTIDOS POR MEIO DA PLATAFORMA SPECIESLINK E A PARTIR DE REVISÃO DE LITERATURA NO PERÍODO DE 2000

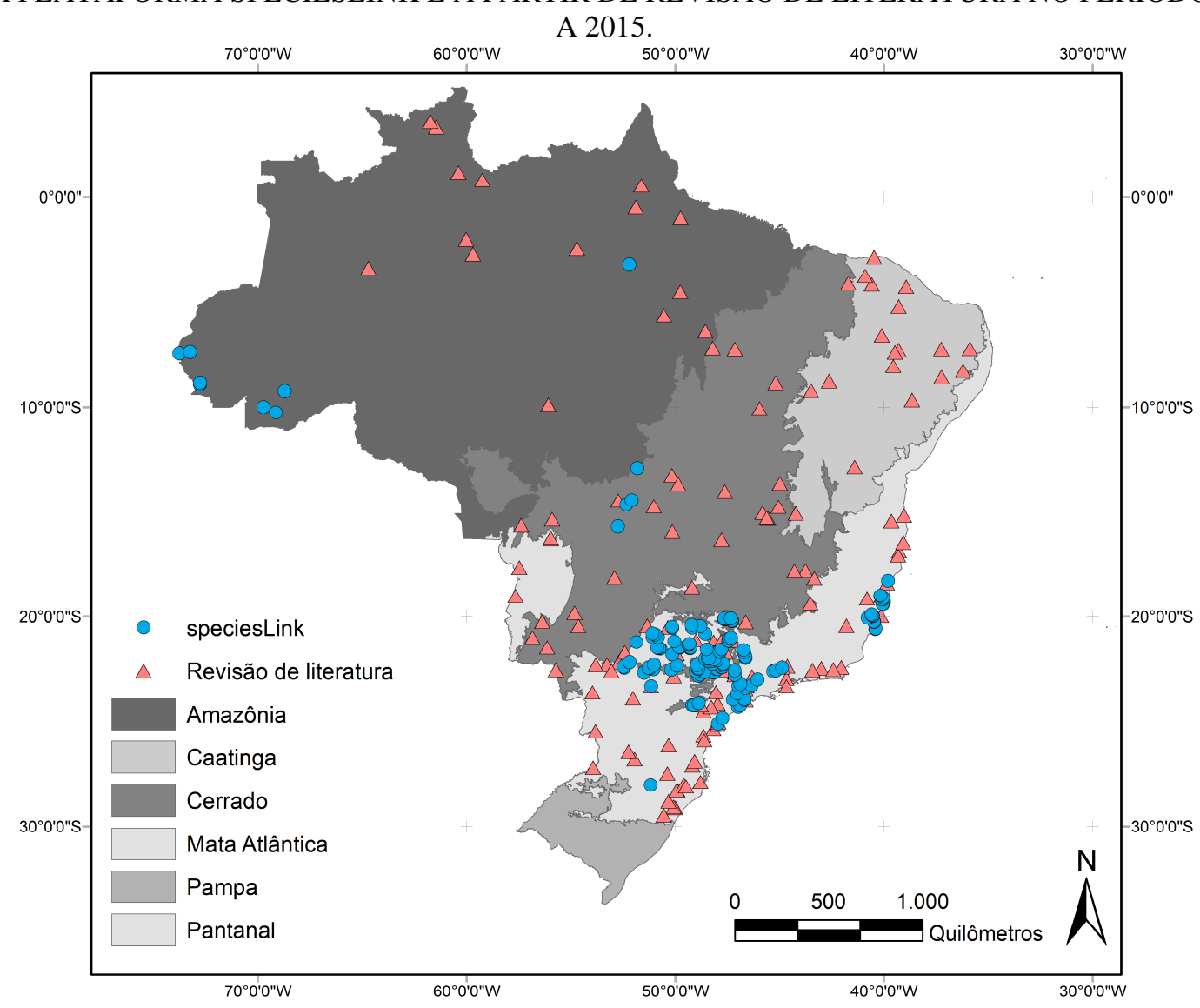

Fonte: O autor (2015)

As análises de colinearidade indicaram forte correlação entre as seguintes variáveis abióticas: bio_5, bio_6, bio_7, bio_8, bio_9, bio_10, bio_11, bio_16 e bio_17 (Figura 2). A partir da análise dessa matriz de correlação, portanto, foram selecionadas para as modelagens as variáveis bio_1, bio_2, bio_3, bio_4, bio_12, bio_13, bio_14, bio_15, bio_18 e bio_19. 
FIGURA 2 - MATRIZ DE CORRELAÇÃO DAS VARIÁVEIS ABIÓTICAS PRÉ-SELECIONADAS A PARTIR DO WORLDCLIM. VARIÁVEIS COM COEFICIENTES DE CORRELAÇÃO SUPERIORES A 0,75 E INFERIORES A 1 ESTÃO ASSINALADAS EM LARANJA. CÓDIGOS DAS VARIÁVEIS CONFORME TABELA 1.

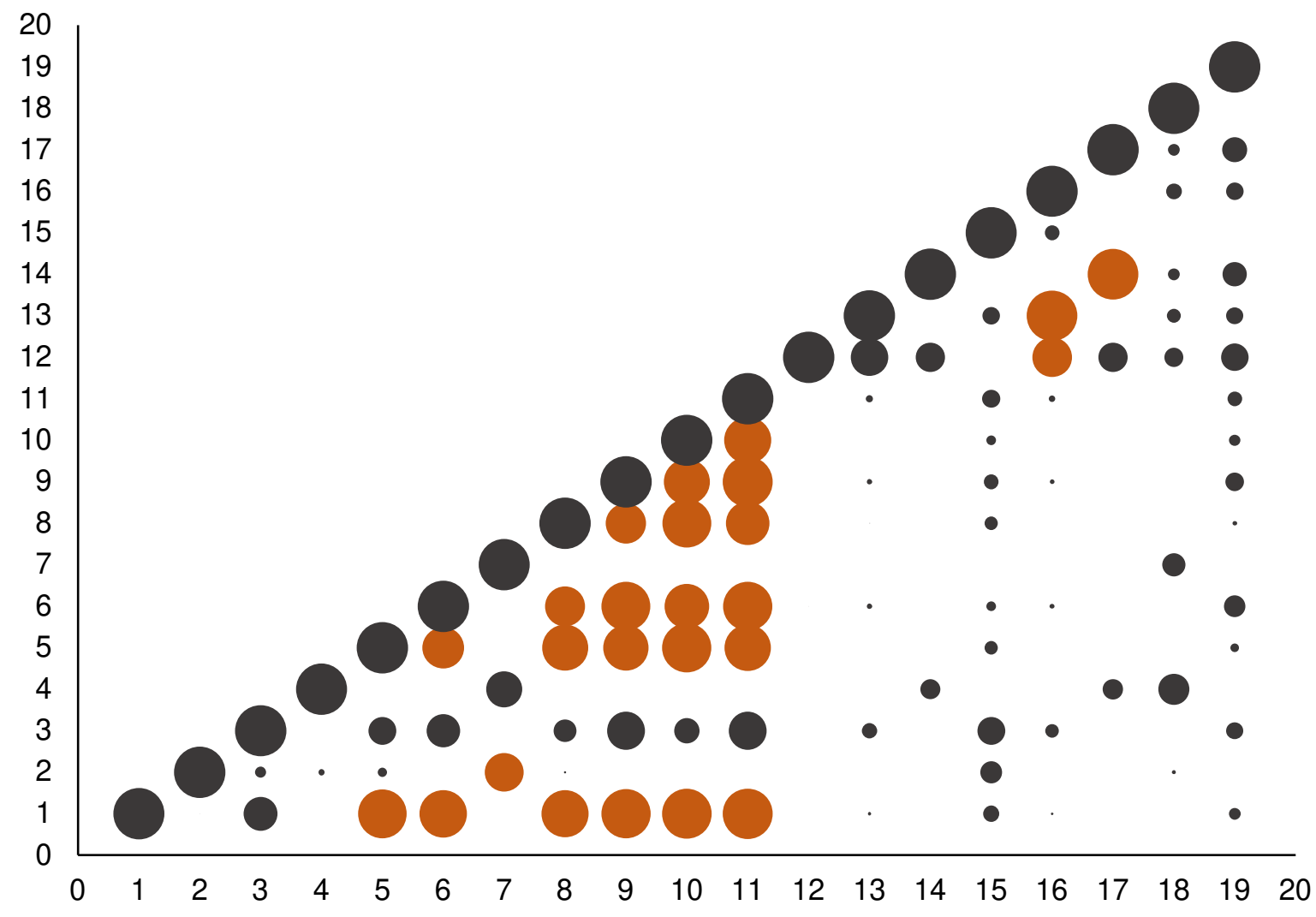

Fonte: O autor (2021).

A modelagem de distribuição de $P$. concolor por meio das variáveis selecionadas através do algoritmo Environmental Distance (Figura 3A) indica que as áreas com maior adequabilidade ambiental e, consequentemente, maior probabilidade de ocorrência de $P$. concolor, se concentram sobretudo na Amazônia ao mesmo tempo em que essa probabilidade decresce na medida em que se avança em direção ao Cerrado, Caatinga e Mata Atlântica. 
FIGURA 3 - MAPAS DE DISTRIBUIÇÃO POTENCIAL DE P. CONCOLOR NO BRASIL. ALGORITMOS UTILIZADOS NA MODELAGEM: (A) MAXENT; (B) BIOCLIM; (C) GARP; (D) ENVIRONMENTAL

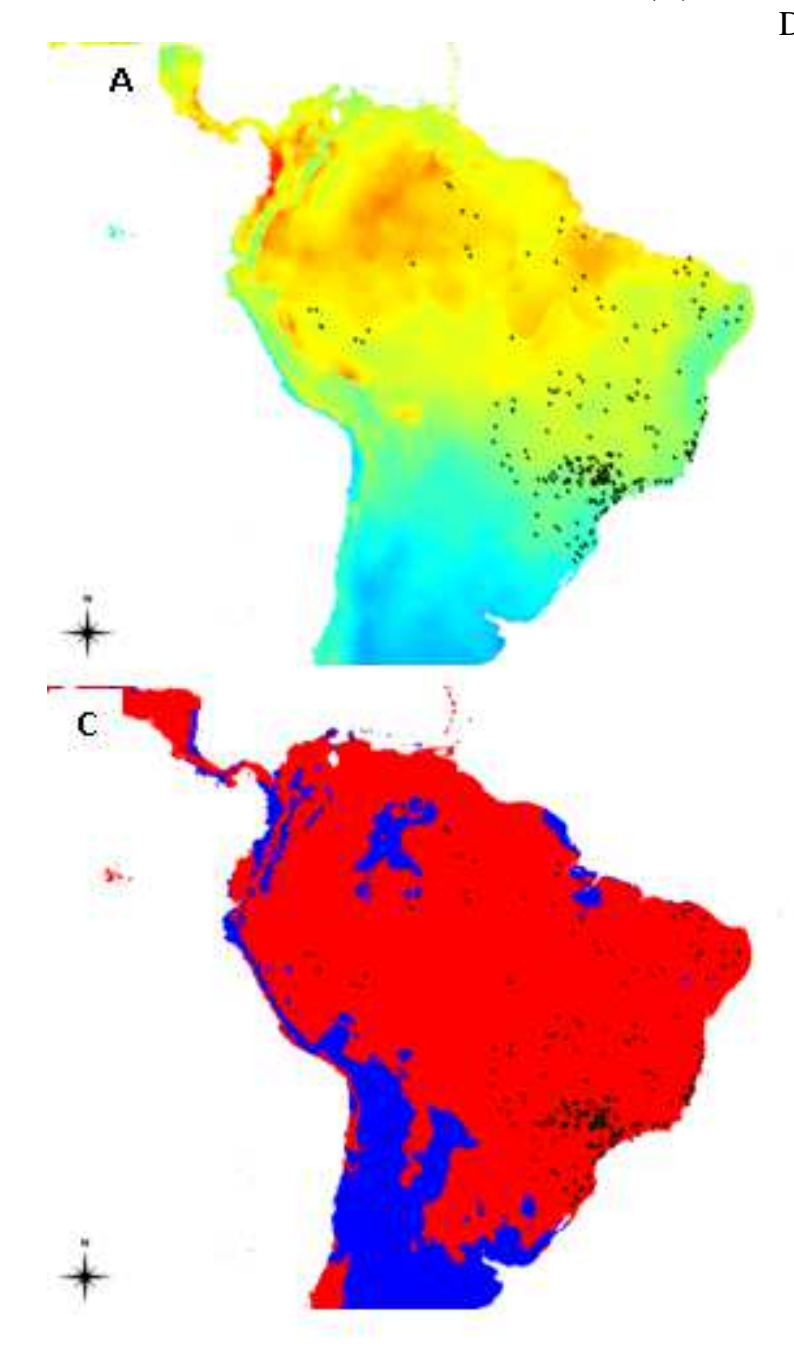
DISTANCE.

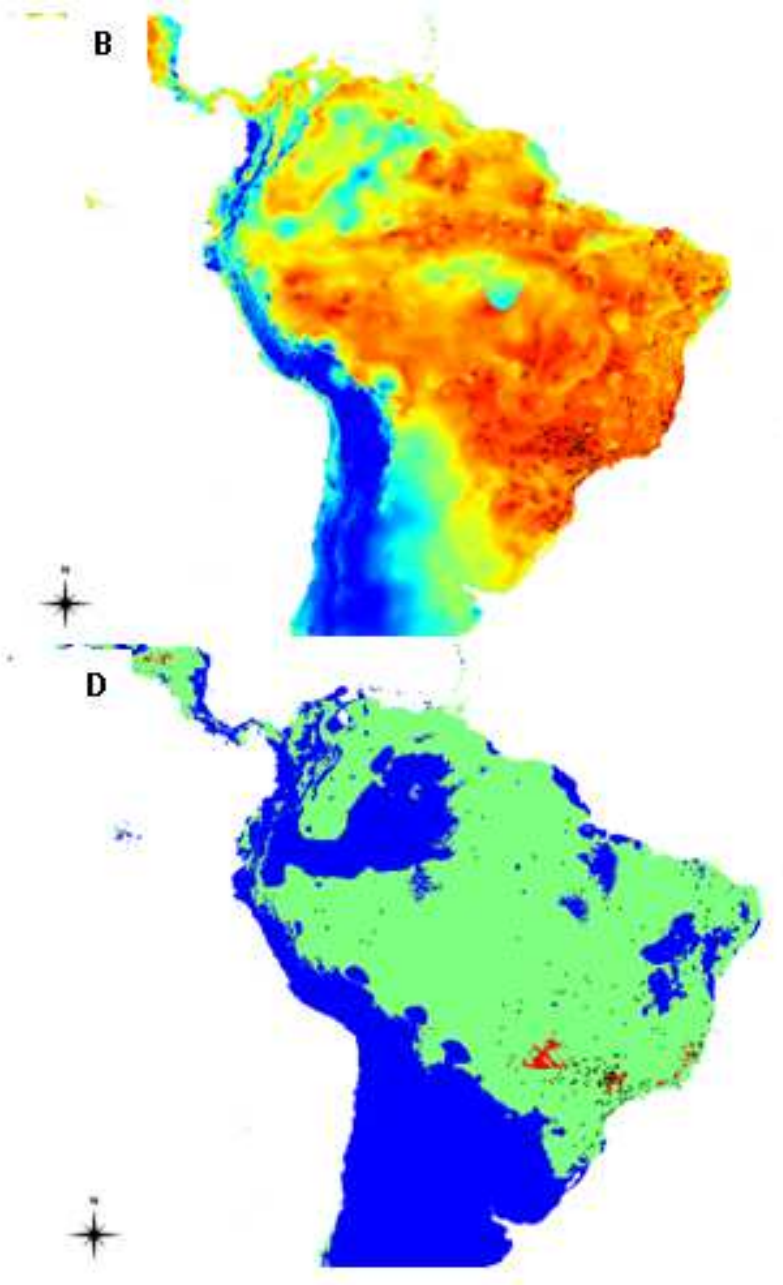

Baixa probabilidade

Alta probabilidade

Fonte: O autor (2015).

Quando modelados a partir do algoritmo Bioclim (Figura 3B) as regiões de maior adequabilidade se distribuíram de forma equivalente em todos os biomas. Entretanto, o mapa gerado sugere menor probabilidade de ocorrência de $P$. concolor em uma região restrita ao sul da Amazônia e das bacias dos rios Xingu e Tapajós. Também foram identificadas como regiões de menor adequabilidade ambiental a porção de Mata Atlântica localizada ao norte do rio São Francisco e diferentes áreas localizadas nos domínios do Pampa.

Os modelos de distribuição obtidos a partir do GARP (Figura 3C) indicam que porções restritas do Pampa no extremo sul do país, bem como pequenos trechos de Caatinga e áreas na 
Amazônia setentrional, apresentam baixa adequabilidade ambiental. As demais regiões, que representam a maior parcela das áreas naturais do país, foram qualificadas como de alta probabilidade de ocorrência.

Com relação aos mapas gerados através da modelagem por meio do algoritmo Environmental Distance (Figura 3D), as áreas de baixa adequabilidade se concentram especialmente na Caatinga e na Amazônia ocidental. Além dessas áreas, o Pampa foi apontado como área de baixa probabilidade de ocorrência de $P$. concolor na maior parte de sua extensão. As demais áreas foram consideradas de média adequabilidade, exceto por duas grandes áreas localizadas na região sul do Cerrado e na Mata Atlântica a sudeste do Brasil, que apresentaram elevada adequabilidade.

Considerando que todos os valores de AUC foram superiores a 0,80 , pode-se assumir que os modelos estão adequadamente ajustados aos dados obtidos e foram validados pelo conjunto de dados de teste utilizado.

\section{DISCUSSÃO}

A interpretação dos diferentes mapas obtidos sugere uma severa diminuição das áreas ocupáveis pela onça-parda no Brasil. Essas áreas se concentram sobretudo na Caatinga, onde os futuros cenários de mudanças climáticas devem produzir uma paisagem incapaz de sustentar tais populações em médio e longo prazo. Além disso, se somam os impactos ocasionados pela pressão das populações humanas dessas regiões, que interferem através da caça, agricultura e urbanização, as quais resultam na perda de habitat e de indivíduos. Sunquist e Sunquist (2002) destacam que onças pardas perderam ao menos $28 \%$ das suas áreas e, embora essa espécie seja resiliente frente às pressões humanas quando comparada a outros carnívoros, a identificação de áreas adequadas e dos mecanismos que operam determinando essa ocupação é essencial para o estabelecimento de medidas que intensifiquem a conectividade estrutural dos habitats e das metapopulações (DE ANGELO et al, 2011).

Com base nos dados obtidos, averiguamos que o atual cenário de distribuição de $P$. concolor se restringe aos últimos remanescentes de Mata Atlântica localizados na região centro-sul do Brasil. Adicionalmente, a maior parte do Cerrado e Amazônia foram consideradas áreas propícias à ocorrência de $P$. concolor através dos distintos algoritmos de modelagem, indicando que a qualidade e extensão das áreas habitáveis nessas duas regiões são um fator determinante para a maior probabilidade de ocorrência de $P$. concolor registrada. Angelieri et al. (2016) verificou a necessidade 
da gestão de áreas protegidas bem como da restauração e proteção de áreas privadas para a conservação da onça-parda no estado de São Paulo, indicando a presença de vegetação nativa, elevação e densidade de rodovias como os melhores preditores da ocorrência da espécie na área avaliada.

Em contrapartida, as regiões semiáridas da Caatinga predominantes na região nordeste foram, de modo geral, representadas como áreas de menor adequabilidade ambiental para a espécie avaliada. As modelagens confirmaram a ausência de $P$. concolor na maior parte da Caatinga e da Mata Atlântica ao norte do rio São Francisco (Centro de Endemismo Pernambuco). Esse bioma tem sido negligenciado por décadas e a insuficiência de informações sobre a espécie na região, somada a uma forte pressão antrópica, tornam essenciais medidas para a preservação da onça-parda. Além disso, a extinção local de $P$. concolor nessas áreas tem sido eventualmente discutida e confirmada (BEZERRA et al., 2014; FERNANDES-FERREIRA et al., 2015; SILVA JR \& MENDES PONTES, 2008) reforçando a importância dos modelos preditivos na identificação de áreas prioritárias para a conservação. Nessas paisagens, a disponibilidade de água e de vegetação mais densa são fatores críticos para a ocorrência de $P$. concolor, indicando que a sazonalidade é fator determinante para a espécie no semiárido (DIAS et a., 2019).

A persistência de populações mínimas viáveis de $P$. concolor a longo prazo em paisagens que têm sido severamente impactadas está condicionada à manutenção de extensas áreas que conciliem áreas protegidas e os últimos remanescentes preservados (MIOTTO et al., 2007). Essa espécie ocupa áreas que podem superar os $100 \mathrm{~km}^{2}$ (SWEANOR et al., 2000) e, quando à procura de alimento, indivíduos podem se deslocar por até $9 \mathrm{~km}$ por noite (BEIER, 1993), reforçando o papel de grandes áreas para a conservação dessas populações. Avaliando a adequabilidade do habitat para os felídeos gato-maracajá (Leopardus wiedii) e jaguarundi ( $P$. yagouaroundi) através do modelo MAXENT, Espinosa et al. (2018) verificou que P. yagouaroundi apresenta menor dependência de ambientes florestados quando comparada à $L$. wiedii. Considerando as semelhanças entre $P$. concolor e P. yagouaroundi e os resultados aqui obtidos, confirma-se a relação entre a ocorrência da onçaparda mesmo em áreas mais abertas.

A conectividade entre as paisagens é outro fator que merece atenção levando-se em consideração os cenários preditivos aqui obtidos. Uma vez que existe elevado risco de mortalidade de onças pardas em decorrência da fragmentação e urbanização (VICKERS et al., 2015), priorizar a criação de corredores ecológicos entre as áreas protegidas representa a alternativa mais acessível tendo em vista a conservação da espécie. Para tanto, é imprescindível que a implantação de 
infraestrutura viária, o impacto do efeito de borda e a largura dos corredores sejam dimensionados com cautela, uma vez que tais fatores determinam a influência humana sobre o movimento dos animais (FORD et al., 2020).

Santana et al. (2020), por exemplo, enfatiza que além dos fatores decorrentes do impacto humano através da expansão da agricultura bem como da ocorrência de eventos climáticos extremos, o enfraquecimento da política ambiental também se revela um fator crítico particularmente no corredor central da Mata Atlântica. No Cerrado, a criação de um corredor para mamíferos de médio e grande porte proposta por Schwaida et al. (2018), demonstrou que as propriedades rurais desempenham um importante papel para a conectividade e podem ser uma alternativa para assegurar o fluxo gênico entre subpopulações de $P$. concolor.

Numa última análise, a criação de áreas protegidas dimensionadas e aptas a abrigar populações da onça-parda podem assegurar não apenas a conservação dessa espécie, mas também de toda a comunidade de mamíferos de pequeno e médio porte com menores exigências em termos de habitat e área de vida. Nesse contexto, destacamos ainda a necessidade da conservação e estabelecimento de ações de manejo na Caatinga, Pantanal e Pampa de modo a viabilizar a ocorrência da espécie também nesses biomas. A implementação de áreas protegidas, controle do impacto da caça e aumento da conectividade da paisagem (estabelecimento de corredores ecológicos), podem representar os primeiros passos nesse sentido.

\section{REFERÊNCIAS}

ANGELIERI, C.C.S.; ADAMS-HOSKING, C.; de BARROS, K.M.P.M. Using species distribution models to predict potential landscape restoration effects on puma conservation. PLoS One, v. 11, n. $1,2016$.

BEIER, P. Determining minimum habitat areas and habitat corridors for cougars. Conservation Biology, v. 7, n. 1, p. 94-108, 1993.

BEZERRA, A. M.; LAZAR, A.; BONVICINO, C. R. \& CUNHA, A. S. Subsidies for a poorly known endemic semiarid biome of Brazil: non-volant mammals of an eastern region of Caatinga. Zoological Studies, v. 53, n. 1, p. 16, 2014.

BUENO, B. A. A. Modelagem de nicho ecológico aplicada à conservação do pato mergulhão (Mergus octosetaceus, Vieillot, 1817) no Brasil. Dissertação: Mestrado em Ecologia e Conservação de Recursos Naturais. Universidade Federal de Uberlândia. 113 p. 2012.

CARPENTER, G.; GILLISON, A. N.; WINTER, J. DOMAIN: a flexible modelling procedure for mapping potential distributions of plants and animals. Biodiversity \& Conservation, v. 2, n. 6, p. 667-680, 1993. 
CRAWSHAW, P.; QUIGLEY, H. A ecologia do Jaguar ou onça pintada no Pantanal. Estudos Bioecológicos do Pantanal. Instituto Brasileiro de Desenvolvimento Florestal (IBDF)/DN (Brasília), 1984.

CRIA. 2015. SpeciesLink. Centro de Referência em Informação Ambiental, Campinas. Electronic Database accessible at http://www.splink.org.br/index. Acessado em 15 nov 2015.

CURRIER, M. J. P. Felis concolor. Mammalian species, p. 1-7, 1983.

CUYCKENS, G. A. E.; PEROVIC, P. G.; CRISTOBAL, L. How are wetlands and biological interactions related to carnivore distributions at high altitude?. Journal of Arid Environments, v. 115, p. 14-18, 2015.

DE ANGELO, C.; PAVIOLO, A.; DI BITETTI, M. Differential impact of landscape transformation on pumas (Puma concolor) and jaguars (Panthera onca) in the Upper Paraná Atlantic Forest. Diversity and Distributions, v. 17, n. 3, p. 422-436, 2011.

DIAS, D. D. M., LIMA MASSARA, R., de CAMPOS, C. B., \& RODRIGUES, F. H. G.. Human activities influence the occupancy probability of mammalian carnivores in the Brazilian Caatinga. Biotropica, v. 51, n. 2, p. 253-265, 2019.

EISENBERG, John F. The density and biomass of tropical mammals. Conservation biology: an evolutionary-ecological perspective, p. 35-55, 1980.

ELITH, J.; PHILLIPS, S. J.; HASTIE, T.; DUDÍK, M.; CHEE, Y. E. \& YATES, C. J. A statistical explanation of MaxEnt for ecologists. Diversity and Distributions, v. 17, n. 1, p. 43-57, 2011.

ENGLER, R.; GUISAN, A.; RECHSTEINER, L. An improved approach for predicting the distribution of rare and endangered species from occurrence and pseudo-absence data. Journal of Applied Ecology, v. 41, n. 2, p. 263-274, 2004.

ESPINOSA, C. C; TRIGO, T. C.; TIRELLI, F. P.; SILVA, L. G.; EIZIRIK, E.; QUEIROLO, D.; MAZIM, F. D.; PETERS, F. B.; FAVARINI, M. O.; FREITAS, T. R. O. de. Geographic distribution modeling of the margay (Leopardus wiedii) and jaguarundi (Puma yagouaroundi): a comparative assessment. Journal of Mammalogy, v. 99, n. 1, p. 252-262, 2018.

FENG, X.; PAPEŞ, M. Ecological niche modelling confirms potential north-east range expansion of the nine-banded armadillo (Dasypus novemcinctus) in the USA. Journal of Biogeography, v. 42, n. 4, p. 803-807, 2015.

FERNANDES-FERREIRA, H.; GURGEL-FILHO, N. M.; FEIJÓ, A.; MENDONÇA, S. V.; DA NÓBREGA ALVES, R. R. \& LANGGUTH, A. Non-volant mammals from Baturité Ridge, Ceará state, Northeast Brazil. Check List, v. 11, n. 3, p. 1630, 2015.

FOLEY, R. A reconsideration of the role of predation on large mammals in tropical hunter-gatherer adaptation. Man, p. 393-402, 1982.

FORD, A. T.; SUNTER, E. J.; FAUVELLE, C.; BRADSHAW, J. L.; FORD, B.; HUTCHEN, J.; PHILLIPOW, N.; TEICHMAN, K. J. Effective corridor width: linking the spatial ecology of wildlife with land use policy. European Journal of Wildlife Research, v. 66, n. 4, p. 1-10, 2020.

GIANNINI, Tereza Cristina. Desafios atuais da modelagem preditiva de distribuição de espécies. Rodriguésia-Instituto de Pesquisas Jardim Botânico do Rio de Janeiro, v. 63, n. 3, 2012.

GITTLEMAN, J. L.; HARVEY, Paul H. Carnivore home-range size, metabolic needs and ecology. Behavioral Ecology and Sociobiology, v. 10, n. 1, p. 57-63, 1982. 
GUISAN, A.; THUILLER, W. Predicting species distribution: offering more than simple habitat models. Ecology letters, v. 8, n. 9, p. 993-1009, 2005.

HUTCHINSON, G. E. Concluding remarks. Cold Spring Harbor Symposia on Quantitative Biology, 22:415-427, 1957.

MIOTTO, R. A.; RODRIGUES, F. P.; CIOCHETI, G., \& GALETTI, P. M. Determination of the minimum population size of pumas (Puma concolor) through fecal DNA analysis in two protected Cerrado areas in the Brazilian southeast. Biotropica, v. 39, n. 5, p. 647-654, 2007.

MORENO, Ricardo S.; KAYS, Roland W. \& SAMUDIO JR, Rafael. Competitive release in diets of ocelot (Leopardus pardalis) and puma (Puma concolor) after jaguar (Panthera onca) decline. Journal of Mammalogy, v. 87, n. 4, p. 808-816, 2006.

NIX, H. A. A biogeographic analysis of Australian elapid snakes. Atlas of elapid snakes of Australia, v. 7, p. 4-15, 1986.

PETERSON, A. T.; SOBERÓN, J.; PEARSON, R. G.; ANDERSON, R.; MARTÍNEZ-MEYER, E.; NAKAMURA, M.; ARAÚJO, M. Ecological Niches and Geographic Distributions. Princeton University Press, Princeton, 2011.

PHILLIPS, S. J.; ANDERSON, R. P.; SCHAPIRE, R. E. Maximum entropy modeling of species geographic distributions. Ecological modelling, v. 190, n. 3, p. 231-259, 2006.

PHILLIPS, Steven J.; DUDÍK, Miroslav. Modeling of species distributions with Maxent: new extensions and a comprehensive evaluation. Ecography, v. 31, n. 2, p. 161-175, 2008.

ROBERGE, JEAN-MICHEL; ANGELSTAM, P. E. R. Usefulness of the umbrella species concept as a conservation tool. Conservation Biology, v. 18, n. 1, p. 76-85, 2004.

SANTANA, R. O.; DELGADO, R. C.; SCHIAVETTI, A. The past, present and future of vegetation in the Central Atlantic Forest Corridor, Brazil. Remote Sensing Applications: Society and Environment, v. 20, p. 100357, 2020.

SCHWAIDA, S. F.; CICERELLI, R. E.; ALMEIDA, T. D. \& ROIG, H. L. Challenges and strategies on implementing an ecological corridor between protected areas in Cerrado biome. Revista Árvore, v. 41, n. 6, 2018.

SCOGNAMILLO, D.; MAXIT, I. E.; SUNQUIST, M. \& POLISAR, J. Coexistence of jaguar (Panthera onca) and puma (Puma concolor) in a mosaic landscape in the Venezuelan llanos. Journal of Zoology, v. 259, n. 03, p. 269-279, 2003.

SILVA JR, A. P.; MENDES PONTES, A. R. The effect of a mega-fragmentation process on large mammal assemblages in the highly-threatened Pernambuco Endemism Centre, north-eastern Brazil. Biodiversity and conservation, v. 17, n. 6, p. 1455-1464, 2008.

SUNQUIST, M. \& SUNQUIST, F. Wild cats of the world. University of Chicago Press, 2002.

STOCKWELL, D. R.B.; NOBLE, I. R. Induction of sets of rules from animal distribution data: a robust and informative method of data analysis. Mathematics and computers in simulation, v. 33, n. 5, p. 385-390, 1992.

STOCKWELL, D. R.B. Genetic algorithms II. In: Machine learning methods for ecological applications. Springer US, 1999. p. 123-144. 
STOCKWELL, D.; PETERS, D. P. The GARP modelling system: problems and solutions to automated spatial prediction. International Journal of Geographical Information Science, v. 13, n. 2, p. 143-158, 1999.

SWEANOR, L. L.; LOGAN, K. A.; HORNOCKER, M. G. Cougar dispersal patterns, metapopulation dynamics, and conservation. Conservation Biology, v. 14, n. 3, p. 798-808, 2000.

TERBORGH, J. The big things that run the world - a sequel to EO Wilson. Conservation Biology, v. 2, n. 4, p. 402-403, 1988.

TERBORGH, J. Maintenance of diversity in tropical forests. Biotropica, p. 283-292, 1992.

TERBORGH, J. The fate of tropical forests: a matter of stewardship. Conservation biology, v. 14, n. 5, p. 1358-1361, 2000.

THORN, J. S.; NIJMAN, V.; SMITH, D.; NEKARIS, K. A. I. Ecological niche modelling as a technique for assessing threats and setting conservation priorities for Asian slow lorises (Primates: Nycticebus). Diversity and Distributions, v. 15, n. 2, p. 289-298, 2009.

VICKERS, T. W.; SANCHEZ, J. N.; JOHNSON, C. K.; MORRISON, S. A.; BOTTA, R.; SMITH, T.; COHEN, B. S.; HUBER, P. R.; ERNEST, H. B.; BOYCE, W. M. Survival and mortality of pumas (Puma concolor) in a fragmented, urbanizing landscape. PloS one, v. 10, n. 7, p. e0131490, 2015. 